\title{
Research Note \\ Radial velocities of giant $M$ stars near the ecliptic ${ }^{\star}$
}

\author{
D. Bettoni ${ }^{1}$ and G. Galletta ${ }^{2}$ \\ 1 Osservatorio Astronomico di Padova, Vicolo dell'Osservatorio 5, 35122 Padova, Italy \\ 2 Dipartimento di Astronomia, Università di Padova, Vicolo dell'Osservatorio 2, 35122 Padova, Italy
}

Received 8 January 2001 / Accepted 18 January 2001

\begin{abstract}
A search dedicated to the identification of new, dwarf and cold stars close to the Sun has allowed us to measure the radial velocities of a set of stars of late spectral type. The stars selected were M-type stars present in the Hipparcos Input Catalogue located in a strip of $\pm 20^{\circ}$ around the Ecliptic and observables from La Silla. Our final set contains 22 M-type stars whose radial velocities $V_{\mathrm{r}}$ have been measured with an accuracy of $\sim 0.5 \mathrm{~km} \mathrm{~s}^{-1}$ during a single observing run. From their velocities, parallaxes and magnitudes we deduce that all the stars observed are red giants, with space velocities relative to the Sun mostly lower than $100 \mathrm{~km} \mathrm{~s}^{-1}$.
\end{abstract}

Key words. catalogues - stars: kinematics - stars: late type - techniques: radial velocities

\section{Introduction}

This research note presents the values of radial velocities measured for a selected sample of 22 stars of spectral type M, extracted from the Hipparcos catalogue (ESA 1997).

The original goal of these observations was the search for a dwarf star very close to the Sun and gravitationally bound to it. The search for such a star was entered in a wider set of studies devoted in the past years to the problem of "Nemesis", a presumed companion of our Sun which is the origin of secular perturbations of the Oort Cloud (see Davis et al. 1984, for details). The discussion of the paleontological implications of these observations have been published elsewhere (Galletta \& Tomadini 1996).

Actually, this goal gave us the opportunity to study several $M$ stars lying in a strip of \pm 20 degrees around the ecliptic. The precision gained by these observations and the lack of radial velocities in the present literature make them still interesting, to increase the number of late sequence stars for which the three components of the velocity vector are known.

Send offprint requests to: G. Galletta,

e-mail: galletta@pd.astro.it

* Based on observations collected at the CAT telescope, European Southern Observatory, La Silla, Chile.

\section{Observations}

The acquisition of the high resolution spectra for the candidate M stars in our list was made at La Silla (Chile). We used the $1.5 \mathrm{~m}$ CAT telescope with the Cassegrain Echelle Spectrograph (CES). The configuration chosen was the Long Camera and a CCD giving a final dispersion of $0.003 \mathrm{~nm} / \mathrm{pix}$ in the region near $\mathrm{H} \alpha$. The wavelength region studied ranged from 653.2 to $658.8 \mathrm{~nm}$. The spectra were obtained in remote observing mode from ESOGarching during two observing nights with exposure times of 5 to $30 \mathrm{~min}$. The signal-to-noise obtained in each spectrum was checked immediately after the acquisition and the exposure was eventually repeated in the same or in the next night, to improve the radial velocity measurements; unfortunately technical problems arising in the third and last night prevented us to get an equal signal-to-noise for all the spectra and the final values range from $S / N=10$ to about 40 in the best cases.

Six spectra of three stars of known radial velocity were taken, to be used as references for the radial velocity measurement. They are HD 44131, HD 51250 and SAO 76297. The first two stars have $V$ magnitude around 5, while the last one is of magnitude 9 . They were extracted from the ESO list of radial velocity standards, mainly derived from IAU Trans. (1955), and are classified gM1, M0 and M5 respectively. Their quoted radial velocities are equal to $+47.4 \pm 0.3 \mathrm{~km} \mathrm{~s}^{-1},+19.6 \pm 0.5 \mathrm{~km} \mathrm{~s}^{-1}$ and $-86.8 \pm 0.5 \mathrm{~km} \mathrm{~s}^{-1}$ respectively. 
A check of the more recent literature indicates a revision for the errors for HD 44131 and HD 51250, that are increased to $\pm 0.9 \mathrm{~km} \mathrm{~s}^{-1}$, while no recent observations have been made for SAO 76297 (Evans 1967; Ochsenbein et al. 2000). Particular care was taken in using HD 51250, as discussed later, because it is part of a quadruple system, as reported by Dommanget \& Nys (1994).

\section{Data reductions}

The spectra were calibrated at ESO with the standard MIDAS package and later reduced in Padova by means of the cross-correlation package of IRAF using the six reference spectra. The velocities were finally corrected to the Sun and to the Local Standard of Rest using the IRAF procedures.

Different fitting functions were used in the cross correlation, i.e. the line barycenter, the Gauss and the Lorentz functions, to take into account the eventual asymmetries of the spectral lines. The results obtained for each couple of spectra (stars vs. standard) gives results mutually consistent within $\pm 0.4 \mathrm{~km} \mathrm{~s}^{-1}$.

In the cases where more than one spectrum was available for the same star, the velocity differences between spectra were found to be always lower than $0.7 \mathrm{~km} \mathrm{~s}^{-1}$, with a mean error of $0.3 \mathrm{~km} \mathrm{~s}^{-1}$.

Finally, the values determined according to the three different standards were compared, showing an agreement within $0.4 \mathrm{~km} \mathrm{~s}^{-1}$, except for the velocities obtained using HD 51250, which produces a systematic shift of $-1.5 \mathrm{~km} \mathrm{~s}^{-1}$. Remembering that this star is member of a multiple system (Dommanget \& Nys 1994), we decided to correct its assumed radial velocity by this shift, which becomes, from our spectra, $18.1 \pm 0.2 \mathrm{~km} \mathrm{~s}^{-1}$. As a check, we also recalculated the radial velocity of the remaining two standard stars, which was $47.4 \pm 0.1 \mathrm{~km} \mathrm{~s}^{-1}$ for HD 44131 and $-87.3 \pm 0.2$ for SAO 76297. The differences to the assumed values from the literature are $\leq 0.5 \mathrm{~km} \mathrm{~s}^{-1}$.

The final radial velocities measured for the sample stars are listed in Table 1, with their errors deduced from different spectra and fitting functions.

\section{Summary}

The sample of 22 M-type stars was observed with a precision better than $0.5 \mathrm{~km} \mathrm{~s}^{-1}$ and their radial velocities reported in Table 1 . Both the velocities corrected to the Sun and to the Local Standard of Rest are quoted. The errors reported near $V_{\text {Sun }}$ are the standard deviation of velocities determined using the three different standard stars.

In agreement with the current bibliography extracted from Ochsenbein et al. (2000), no star listed in Table 1 is known to be binary. However, we note that the velocities comes from 1-epoch observations, and may be influenced by the presence of a non-detected companion.
Table 1. Radial velocity determinations for the sample stars. Values corrected to the Sun $\left(V_{\text {Sun }}\right)$ and to the Local Standard of Rest $\left(V_{\mathrm{LSR}}\right)$ are quoted. The errors reported near $V_{\text {Sun }}$ are the standard deviations from the velocities determined using different standard stars. A comparison with the values listed in literature for standard stars indicates errors within $0.5 \mathrm{~km} \mathrm{~s}^{-1}$

\begin{tabular}{rrrr}
\hline HIP & SAO & \multicolumn{1}{c}{$V_{\text {Sun }}$} \\
& & $\begin{array}{r}V_{\text {LSR }} \\
\mathrm{km} \mathrm{s}^{-1}\end{array}$ \\
\hline 989 & 73809 & $-11.28 \pm 0.15$ & -8.18 \\
1497 & 91838 & $-32.64 \pm 0.22$ & -31.75 \\
3518 & 109427 & $6.62 \pm 0.20$ & 3.82 \\
6220 & 109810 & $-2.86 \pm 0.25$ & -7.59 \\
8196 & 129485 & $3.32 \pm 0.08$ & -4.91 \\
12468 & 75509 & $57.74 \pm 0.23$ & 51.00 \\
12731 & 93073 & $24.24 \pm 0.16$ & 16.47 \\
15089 & 130374 & $53.45 \pm 0.07$ & 39.97 \\
16728 & 111276 & $82.09 \pm 0.21$ & 68.36 \\
17379 & 93566 & $58.26 \pm 0.31$ & 46.45 \\
21633 & 111950 & $29.87 \pm 0.25$ & 14.78 \\
26786 & 94760 & $53.30 \pm 0.03$ & 39.39 \\
103769 & 145037 & $-40.88 \pm 0.17$ & -29.56 \\
105575 & 145352 & $38.02 \pm 0.23$ & 47.78 \\
107007 & 145575 & $20.01 \pm 0.05$ & 28.77 \\
108298 & 145758 & $-23.00 \pm 0.13$ & -15.93 \\
108844 & 145834 & $-60.99 \pm 0.20$ & -52.48 \\
111365 & 127600 & $-35.05 \pm 0.25$ & -28.46 \\
114053 & 127964 & $-63.74 \pm 0.10$ & -58.10 \\
114608 & 128037 & $7.00 \pm 0.10$ & 12.12 \\
115657 & 165684 & $-25.37 \pm 0.21$ & -24.76 \\
116223 & 128240 & $7.20 \pm 0.10$ & 9.88 \\
\hline & & &
\end{tabular}

From the values listed, we see that no close, dwarf stars are present; all the stars appear to be red giants, with absolute $V$ magnitude ranging from $\sim+2$ to -3 , and located at distances from the Sun greater than 195 pc. A short analysis of the values listed in the Table also shows that all listed stars have space velocities relative to Sun $<130 \mathrm{~km} \mathrm{~s}^{-1}$.

Acknowledgements. We made use of the Vizier service (Ochsenbein et al. 2000) for data collection from various catalogues.

\section{References}

Davis, M., Hut, P., \& Muller, R. A. 1984, Nature, 308, 715

Dommanget, J., \& Nys, O. 1994, Comm. Obs. Royal de Belgique, 115, 1

Evans, D. S. 1967, IAU Symp. 30, 57

Galletta, G., \& Tomadini, L. 1996, in Reports of Shallow Tethys IV, Ann. Mus. Civ. Rovereto, ed. E. KristanTollmann, \& G. Piccoli, 11, 7

The Hipparcos and Tycho Catalogues (vols. 1-17), 1997, European Space Agency SP-1200

Trans. IAU (1955) 9, 442

Ochsenbein, F., Bauer, P., \& Marcout, J. 2000, A\&AS, 143, 23

Turon, C., et al. The Hipparcos Input Catalogue, Version 2, European Space Agency SP-1136 